\title{
AUTORÍA FEMENINA EN LA REVISTA PERUANA DE MEDICINA EXPERIMENTAL Y SALUD PÚBLICA: ANÁLISIS DEL PERIODO 1997-2017
}

\author{
Dylan García-Aguilar ${ }^{1, a}$, Isabel Heredia-Mimbela ${ }^{2, b}$, Reneé Pereyra-Elías $1, \mathrm{c}$
}

\begin{abstract}
RESUMEN
Objetivos. Explorar la participación femenina en la autoría de los artículos publicados en la Revista Peruana de Medicina Experimental y Salud Pública entre 1997 y 2017, y su asociación con características del equipo de investigación. Materiales y métodos. Estudio bibliométrico y analítico. Se evaluaron las tendencias en el tiempo de varias formas de participación femenina, incluyendo «roles privilegiados» de autoría (primer autor, autor corresponsal y autor senior), así como la proporción de mujeres respecto al total de autores. Se comparó la participación femenina de acuerdo a la participación de médicos(as) y autores de filiación extranjera. Resultados. Se analizaron 1606 artículos, 46,3\% fueron originales/originales breves. El $63,5 \%$ tuvieron al menos una autora, mientras que $92,8 \%$ tuvieron al menos un autor varón. El $29,6 \%, 27,8 \%$ y $26,9 \%$ tuvieron a una mujer como primer autor, corresponsal o senior, respectivamente; $33 \%$ de autores por artículo fueron mujeres. Estas cifras variaron durante el periodo de estudio, pero no mostraron diferencias entre el inicio y el final del mismo. En artículos originales, la participación femenina fue menor si quien ocupaba los roles privilegiados de autoría era un médico(a) y mayor si era un autor de filiación extranjera. Los tiempos editoriales fueron mayores cuando había una mujer en una posición privilegiada de autoría. Conclusiones. Menos de un tercio de los artículos tuvo a una mujer en alguno de los roles privilegiados de autoría. Estas brechas, aparentemente, no han mejorado con el tiempo. La composición del equipo de investigación influye en la participación femenina en la autoría.
\end{abstract}

Palabras clave: Mujeres; Autoría y Coautoría en la Publicación Científica; Publicaciones; Perú (fuente: DeCS BIREME).

\section{FEMALE AUTHORSHIP IN THE REVISTA PERUANA DE MEDICINA EXPERIMENTAL Y SALUD PUBLICA: ANALYSIS FOR THE PERIOD 1997-2017}

\begin{abstract}
Objectives. To explore female authorship trends among the articles published in the Revista Peruana de Medicina Experimental y Salud Pública between 1997 and 2017, as well as its association with characteristics of the research teams. Materials and Methods. We conducted a bibliometric analysis. We evaluated time trends of several forms of female participation in authorship, including "privileged authorship roles" (first author, corresponding author and senior author) and the proportion of female authors (out of the total number of authors). We compared female authorship according to the participation of medical doctors and authors from non-Peruvian institutions. Results. We analysed $1606 / 1621$ articles; $46.3 \%$ were original articles. $63.5 \%$ had at least one female author, whilst $92.8 \%$ had at least one male author. A woman was the first, corresponding or senior author in $29.6 \%, 27.8 \%$ and $26.9 \%$ of the articles, respectively. $33 \%$ of authors per article were female (median). These figures did not considerably vary between the start and end of the study period. For original articles, female authorship was less likely if medical doctors were listed in the privileged authorship roles and more likely, if authors from non-Peruvian institutions were. Editorial processing times were longer when a woman served a privileged authorship role. Conclusions. Women served privileged authorship roles in less than a third of all articles. Apparently, these gaps have not improved over time. The composition of the research team predicts female authorship.
\end{abstract}

Keywords: Women; Authorship; Authorship and Co-Authorship in Scientific Publications; Publications; Peru. (source: MeSH NLM).

\footnotetext{
Escuela de Medicina, Universidad Peruana de Ciencias Aplicadas. Lima, Perú.

Área de Humanidades, Universidad Peruana de Ciencias Aplicadas. Lima, Perú.

Médico cirujano; ${ }^{b}$ licenciada en Educación, maestro en Educación; ; ${ }^{c}$ médico cirujano, maestro en Epidemiología.

Los resultados del presente estudio fueron presentados en forma de póster en el "IV Encuentro de Científicos Peruanos en Europa - SINAPSIS", Ghent, Bélgica, 2019.

Recibido: 23/04/2019 Aprobado: 04/09/2019 En línea: 03/12/2019
}

Citar como: García-Aguilar D, Heredia-Mimbela I, Pereyra-Elías R. Autoría femenina en la Revista Peruana de Medicina Experimental y Salud Pública: análisis del periodo 1997-2017. Rev Peru Med Exp Salud Publica. 2019;36(4):601-9. doi: 10.17843/rpmesp.2019.364.4488. 


\section{INTRODUCCIÓN}

La participación de la mujer en las ciencias biomédicas se ha incrementado con el paso de los años. En la década de los sesenta, menos del $10 \%$ de estudiantes de medicina era mujer ${ }^{(1)}$. Actualmente, reportes de distintas latitudes muestran que la proporción de mujeres se encuentra alrededor del $50 \%$ o es incluso más alta ${ }^{(1-5)}$. En Estados Unidos, de acuerdo con la Association of American Medical Colleges (AAMC), tanto varones como mujeres ingresan y egresan de las facultades de medicina en una proporción similar (1). De forma similar, en Latinoamérica y en Perú, las mujeres representan aproximadamente $50 \%$ de los estudiantes de medicina ${ }^{(4)}$. No obstante, no se evidencia un progreso similar en la parte académica de la medicina. Para el 2014, en Estados Unidos, las mujeres ocuparon el $33 \%$ de las posiciones académicas. Asimismo, las mujeres presentaron menor probabilidad de ser profesores principales (Full professor) ${ }^{(6)} \mathrm{y}$, en promedio, tenían un salario menor al de los varones (7). Estas diferencias persistieron después de controlar el efecto de la edad, especialidad y años de experiencia (y posición académica para las diferencias salariales) ${ }^{(6,7)}$.

En cuanto a producción científica, una menor participación femenina en la autoría de artículos científicos en revistas revisadas por pares ha sido documentada por décadas. Sin embargo, estudios internacionales en diversas áreas del conocimiento biomédico han mostrado que esta ha aumentado con el tiempo ${ }^{(8-18)}$. Sin embargo, aún existe una baja representación de mujeres en el rol de primer autor, así como de «autor senion», en el que la brecha es aún mayor (el autor senior es tradicionalmente el miembro más experimentado o jefe del equipo de investigación y es listado como último autor) ${ }^{(8-13,16-17)}$. Además, algunos estudios sugieren que el incremento de la proporción de mujeres en estos roles de autoría ha alcanzado una meseta en los últimos 10 a 15 años ${ }^{(10,11,13,14)}$.

No se disponen de estudios peruanos que evalúen tendencias de autoría femenina en el tiempo. Por ello, el objetivo del presente estudio es explorar la participación femenina en la autoría de los artículos publicados en la Revista Peruana de Medicina Experimental y Salud Pública (RPMESP) entre 1997 y 2017. La RPMESP es la publicación oficial del Instituto Nacional de Salud del Perú y es la única revista biomédica general (miscelánea en temas de ciencias de la salud y salud pública) indizada a Scopus y MEDLINE ${ }^{(19,20)}$. Adicionalmente, se evaluó si la participación femenina se encontraba asociada a ciertas características del equipo de investigación, como la participación de médicos y de autores de filiación extranjera; y si los tiempos editoriales de la revista variaban según participación femenina en la autoría.

\section{MENSAJES CLAVE}

Motivación para realizar el estudio. Globalmente, existe una subrepresentación femenina en la autoría de publicaciones científicas. Este fenómeno no ha sido evaluado en Perú.

Principales hallazgos. Delos artículos publicados entre 1997 y 2017 en la Revista Peruana de Medicina Experimental y Salud Pública, menos de un tercio tuvo a una mujer como primera autora, senior o corresponsal. Estas proporciones, aparentemente, no han mejorado con el tiempo. La participación femenina fue menor en artículos liderados por médicos y mayor en los liderados por autores extranjeros.

Implicancias. Se requiere mayor investigación para entender mejor este fenómeno y crear intervenciones necesarias para cerrar brechas.

\section{MATERIALES Y MÉTODOS}

\section{DISEÑO DE ESTUDIO}

Se realizó un estudio bibliométrico y analítico. Los datos fueron obtenidos de los artículos publicados en la RPMESP. Esta revista se encuentra indizada en SciELO, Scopus, MEDLINE (en estas dos últimas desde el 2010), entre otras bases de datos. Se revisaron todos los artículos publicados en un periodo de 21 años (entre 1997 y 2017), mediante el archivo digital que se encuentra en la página web de la RPMESP (https://rpmesp.ins.gob.pe/index.php/rpmesp/ issue/archive) entre los meses de febrero y marzo de 2018. Los artículos que no consignaron autores individuales fueron excluidos.

\section{VARIABLES}

Se consideró como variable principal, a la participación femenina en la autoría de las publicaciones. Asimismo, se evaluaron otras covariables de interés.

Las distintas formas de participación femenina en la autoría fueron consideradas como: a) Primera autoría femenina, si el primer autor listado es una mujer; b) autoría senior femenina, si el último autor listado es una mujer ${ }^{(8,9,11-13,16,17) \text {; }}$ c) autor corresponsal mujer, si el autor corresponsal -en el que se indique dirección o correo electrónicoes una mujer; d) existencia de al menos una mujer dentro de la lista de autores de la publicación; y e) proporción de autores mujeres (calculado dividiendo el número de autoras entre el número total de autores de la publicación). En las publicaciones con una única autora, se consideró que una mujer cumplía el rol de «primer autor», «autor senion» y «autor corresponsal» simultáneamente. Por facilitar la lectura, donde corresponda, se considera como «primer autor», «autor senion» y «autor corresponsal» como roles/ posiciones de autoría, independientemente de si estos fueran varones o mujeres. Asimismo, la primera autoría, autoría senior y a la corresponsalía, fueron considerados como 
«roles privilegiados de autoría». Cabe precisar que cada tipo de participación femenina descrita fue evaluada y analizada de forma independiente y no forman parte de un constructo que considera todas a la vez.

Asimismo, se evaluaron otras variables, como el tipo de artículo según la clasificación de la RPMESP: editorial, original, original breve, simposio, artículo de revisión, reporte de caso, carta al editor, galería fotográfica, sección especial e historia de la medicina. Además, del año de la publicación, la profesión (médico vs. no-médico) y la afiliación institucional (peruana vs extranjera) del primer autor, autor senior y corresponsal. Finalmente, se evaluó los tiempos de los procesos editoriales: desde el envío a la aceptación, desde la aceptación hasta la publicación y el total desde el envío hasta la publicación (tiempo en días, según lo consignado en los artículos).

\section{PROCEDIMIENTOS}

La determinación del género de los autores se realizó analizando los nombres de pila (primer nombre, así como segundo y tercer nombre cuando estuvieron listados). Esta metodología ha sido utilizada previamente en estudios similares ${ }^{(8-11,14,16)}$. No se contactó por correo electrónico, teléfono, redes sociales (como Facebook) o ninguna otra vía a los autores evaluados.

En caso de que el nombre sea ambiguo, se revisó la página web de su institución académica o de sus cuentas académicas relacionadas (Google Scholar, ResearchGate, LinkedIn) en busca de fotos o párrafos biográficos, tal como se describe en la metodología de un estudio recientemente publicado en The British Medical Journal (10). Sólo se recogieron datos públicamente disponibles. Asimismo, para los autores peruanos, se buscó en la página web del Colegio Médico del Perú (CMP), en el Directorio Nacional de Investigadores e Innovadores (DINA) o en el portal de la Seguridad Social (EsSalud).

La recolección de datos fue realizada por los autores (DGA e IHM) de manera independiente. Las discordancias fueron resueltas por consenso entre los tres autores.

\section{ASPECTOS ÉTICOS}

El estudio fue exonerado de revisión por el Comité de Ética de la Facultad de Ciencias de la Salud de la Universidad Peruana de Ciencias Aplicadas.

\section{ANÁLISIS DE DATOS}

Se realizó doble digitación de datos en Microsoft Excel ${ }^{\circledR}$. Luego del control de calidad, la base de datos fue exportada al paquete estadístico Stata 12.0 (StataCorp, Texas, USA) para su análisis. Se evaluó la proporción de la participación femenina en sus formas descritas. Para el número y proporción de autoras en las publicaciones analizadas, se calculó la mediana de las mismas (acompañada de mínimomáximo y rangos intercuartílicos, respectivamente). Se analizó la tendencia de estas variables a través del tiempo de estudio, el cual fue agrupado en trienios. No realizamos un análisis por año debido a que, en parte del periodo de estudio, el limitado número de publicaciones por año no lo permitía. Estas variables fueron evaluadas en el total de publicaciones y en el subgrupo de artículos originales/ originales breves (formado IMRD - Introducción, Métodos, Resultados y Discusión). Se realizaron gráficos para una apreciación visual de la participación femenina en el tiempo utilizando el programa Microsoft Excel ${ }^{\circledR}$. Asimismo, se aplicó el chi-cuadrado de tendencia para evaluar la existencia de una tendencia lineal en las proporciones de participación femenina.

En el caso de artículos originales/originales breves, se analizó si existían diferencias entre la participación femenina en la autoría según la participación de médicos y de autores extranjeros en los artículos. Esto fue evaluado con las pruebas de chi-cuadrado y suma de rangos de Wilcoxon. Por último, comparamos los tiempos editoriales según participación femenina en la autoría con la prueba de suma de rangos de Wilcoxon y la extensión de Cuzik de la misma para evaluación de tendencia. Se consideró un valor de $p<0,05$ como significativo.

\section{RESULTADOS}

\section{DESCRIPCIÓN GENERAL DE LAS PUBLICACIONES}

En el periodo de estudio, fueron publicados en total 1621 artículos; de los cuales, fueron excluidos 15 , en los que no se listó a los autores de forma individual. La muestra final incluyó 1606 artículos analizados. El número de artículos publicados incrementó con los años (1997-1999: 2,6\%; 2015-2017: 24,7\%). De acuerdo con el tipo de publicación, $588(36,6 \%)$ fueron artículos originales y $155(9,7 \%)$, originales breves. La proporción de cada tipo de artículo en forma global y según trienios se encuentra detallada en la Tabla 1.

\section{PARTICIPACIÓN FEMENINA EN LA AUTORÍA DE LAS PUBLICACIONES}

De todos los artículos evaluados, $62,5 \%$ contó con al menos una mujer en la lista de autoras. Mientras tanto, 92,8\% tuvieron al menos un varón como autor. Aproximadamente, $29,6 \%$ tuvo a una mujer como primera autora, el $27,8 \%$ como autora senior y el $26,9 \%$ como autora corresponsal. La mediana de la proporción de autoras mujeres en las publicaciones fue 33\% (Tabla 1). La participación femenina ha seguido un patrón con ligeras fluctuaciones, con una menor participación hacia la mitad del periodo de estudio, pero con proporciones similares entre el inicio y el final (Tabla 1; Figura 1). En los artículos originales y originales 
Tabla 1. Características y participación femenina en los artículos publicados en la Revista Peruana de Medicina Experimental y Salud Pública entre 1997 y 2017

\begin{tabular}{|c|c|c|c|c|c|c|c|c|c|}
\hline \multirow[t]{2}{*}{ Características } & \multicolumn{2}{|c|}{ Total } & \multirow[t]{2}{*}{$\begin{array}{c}1997-1999 \\
n=41\end{array}$} & \multirow[t]{2}{*}{$\begin{array}{c}2000-2002 \\
n=68\end{array}$} & \multirow[t]{2}{*}{$\begin{array}{c}\text { 2003-2005 } \\
n=143\end{array}$} & \multirow[t]{2}{*}{$\begin{array}{c}2006-2008 \\
n=209\end{array}$} & \multirow[t]{2}{*}{$\begin{array}{c}2009-2011 \\
n=341\end{array}$} & \multirow[t]{2}{*}{$\begin{array}{c}2012-2014 \\
n=407\end{array}$} & \multirow[t]{2}{*}{$\begin{array}{c}2015-2017 \\
n=397\end{array}$} \\
\hline & $\mathbf{n}$ & $\%$ & & & & & & & \\
\hline Total & 1606 & 100 & 2,6 & 4,2 & 8,9 & 13 & 21,2 & 25,4 & 24,7 \\
\hline \multicolumn{10}{|l|}{ Tipo de publicación } \\
\hline Editorial & 87 & 5,4 & 0,0 & 2,9 & 7,0 & 7,2 & 6,2 & 4,9 & 4,8 \\
\hline Original & 588 & 36,6 & 78,1 & 73,5 & 63,6 & 53,6 & 23,8 & 25,3 & 30,0 \\
\hline Original breve & 155 & 9,7 & 0,0 & 0,0 & 0,0 & 0,0 & 14,1 & 11,6 & 15,1 \\
\hline Simposio & 170 & 10,6 & 0,0 & 0,0 & 0,0 & 10,1 & 17,3 & 11,6 & 10,8 \\
\hline Revisión & 95 & 5,9 & 14,6 & 10,3 & 9,8 & 7,7 & 4,7 & 4,7 & 4,3 \\
\hline Reporte de caso & 69 & 4,3 & 2,4 & 1,5 & 4,2 & 3,8 & 3,5 & 5,2 & 5,0 \\
\hline Carta al editor & 265 & 16,5 & 0,0 & 0,0 & 2,8 & 10,1 & 18,8 & 23,3 & 20,4 \\
\hline Galería fotográfica & 48 & 3,0 & 0,0 & 11,8 & 6,3 & 3,4 & 3,2 & 2,2 & 1,0 \\
\hline Sección especial & 94 & 5,9 & 4,9 & 0,0 & 4,9 & 1,4 & 5,0 & 8,6 & 7,6 \\
\hline Historia & 35 & 2,1 & 0,0 & 0,0 & 1,4 & 2,9 & 3,5 & 2,7 & 1,0 \\
\hline \multicolumn{10}{|l|}{ Participación femenina } \\
\hline Primera autora & 476 & 29,6 & 41,5 & 32,4 & 27,3 & 32,5 & 19,9 & 26,3 & 39,0 \\
\hline Autora senior & 447 & 27,8 & 39,0 & 41,2 & 31,5 & 26,3 & 18,8 & 27,3 & 32,2 \\
\hline Autora corresponsal & 423 & 26,9 & 50,0 & 32,8 & 23,2 & 30,6 & 17,6 & 23,3 & 36,3 \\
\hline Al menos una autora & 1004 & 62,5 & 65,9 & 64,7 & 60,8 & 62,2 & 51,9 & 62,7 & 71,5 \\
\hline Número de autores (total)* & 3 & (1 a 26) & $3(1 \mathrm{a} 13)$ & $3(1 \mathrm{a} 10)$ & $3(1$ a 16$)$ & $3(1$ a 16$)$ & $3(1$ a 19$)$ & $3(1$ a 26$)$ & $4(1 \mathrm{a} 15)$ \\
\hline Número de autoras* & 1 & (0 a 16) & $1(0$ a 5$)$ & $1(0$ a 6$)$ & $1(0$ a 8$)$ & $1(0$ a 7$)$ & $1(0$ a 8$)$ & $1(0$ a 16$)$ & $1(0$ a 9$)$ \\
\hline Proporción de autoras ${ }^{\dagger \ddagger}$ & $33 \%$ & $(0$ a 50$)$ & $33(0$ a 60$)$ & 33 (0 a 67) & $33(0$ a 60$)$ & $33(0$ a 50$)$ & $16(0$ a 40) & $33(0$ a 50$)$ & $33(0$ a 50$)$ \\
\hline
\end{tabular}

*Mediana (mínimo, máximo).

†Proporción de autoras entre el número de total de autores del artículo.

‡ Mediana (rango intercuartílico).

breves, la participación femenina en el tiempo fue similar a las publicaciones en general, pero con proporciones ligeramente más altas que en el total de artículos (Figura 1). Las pruebas estadísticas tampoco sugirieron la existencia de una tendencia en el tiempo (valor de $p>0,05$ para todos los tipos de participación femenina en autoría).

En los 282 artículos originales en los que una mujer fue primera autora, $44,0 \%$ de ellas se encontraba afiliada a un centro de investigación, 40,4\% a una universidad, 7,8\% a un hospital y $7,8 \%$ a otro tipo de instituciones. Para los 257 artículos originales con mujeres como autoras

A.

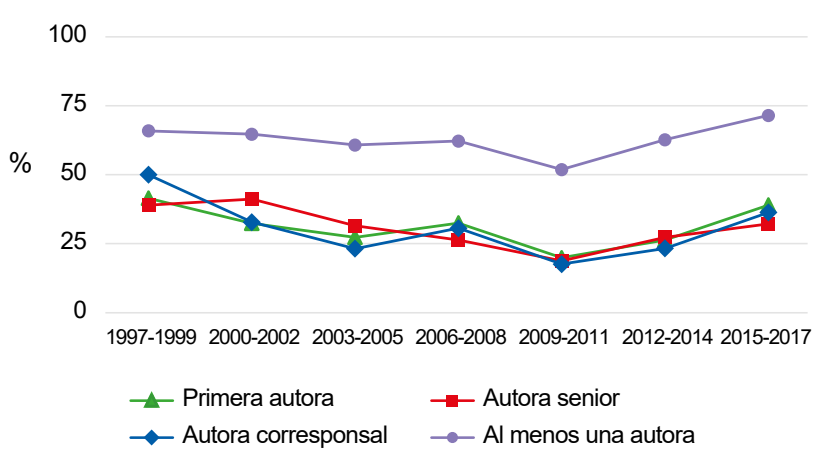

senior, las proporciones para las mismas afiliaciones fueron $35,8 \%, 35,8 \%, 16,0 \%$ y $12,4 \%$. Finalmente, en los 247 artículos con mujeres como autoras corresponsales, las proporciones fueron $45,3 \%, 40,1 \%, 7,7 \%$ y $6,9 \%$, respectivamente.

\section{PARTICIPACIÓN FEMENINA EN LA AUTORIAA SEGÚN LA PROFESIÓN DE LOS INVESTIGADORES}

La proporción de artículos con una mujer como primera autora fue menor si el primer autor era un médico(a) (20,4\% vs. $52,5 \% ; p<0,001)$, si el autor senior era médico(a)

B.

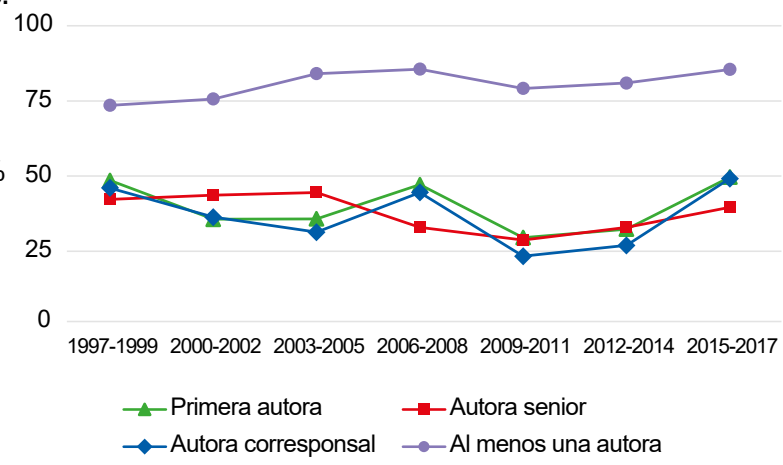

Figura 1. A. Participación femenina en todas las publicaciones y B. Participación femenina en artículos originales (incluye originales breves) de la Revista Peruana de Medicina Experimental y Salud Pública entre 1997 y 2017. 
(34,5\% vs. $41,9 \% ; p=0,039)$ y si el autor corresponsal era médico(a) (23,3\% vs. $50,7 \%$; $p<0,001)$. Similarmente, la probabilidad de encontrar a una mujer en la posición de senior, en la corresponsalía o encontrar al menos una mujer en la lista de autores fue significativamente menor en los artículos en los que un médico(a) fue primer autor, senior o corresponsal. La mediana de la proporción de mujeres como autoras, también, fue menor en estos escenarios (Tabla 2).

\section{PARTICIPACIÓN FEMENINA EN LA AUTORÍA SEGÚN AFILIACIÓN DE INVESTIGADORES}

La proporción de artículos con una mujer como primera autora fue mayor si la filiación del primer autor era de una institución extranjera ( $50,1 \%$ vs. $36,0 \%$; $p=0,006)$, si el autor senior era de una institución extranjera $(46,1 \%$ vs. $36,5 \%$; $p=0,052)$ y si el autor corresponsal era de una institución extranjera $(49,5 \%$ vs. $35,7 \%$; $p=0,008)$. Se observan resultados similares para la participación de una mujer como autora senior y corresponsal. No hubo diferencias considerables entre la proporción de artículos con al menos una autora entre las publicaciones de autores extranjeros o peruanos. La mediana de la proporción de autoras por artículo fue mayor en los tres escenarios de participación extranjera (Tabla 3).

\section{TIEMPOS EDITORIALES SEGÚN PARTICIPACIÓN FEMENINA}

La mediana de tiempo desde el envío del artículo a la revista y su aceptación para publicación fue significativamente mayor si una mujer ocupaba la posición de primer autor (150 días vs. 119 días; $p<0,001$ ), autor senior (143 días vs. 121 días; $p=0,008)$ o autor corresponsal (151 días vs. 119 días; $p<0,001)$. No se observan diferencias entre el tiempo desde la aceptación hasta la publicación de los artículos. El tiempo total desde el envío hasta la publicación fue significativamente mayor cuando las mujeres ocupaban las posiciones de primera autoría (224 días vs. 185 días; $\mathrm{p}=0,002$ ) y corresponsalía (227 días vs. 182 días; $p<0,001$ ). Asimismo, mientras mayor la proporción de autoras, mayor la mediana de los tiempos editoriales (Tabla 4).

\section{DISCUSIÓN}

Este estudio buscó evaluar la participación femenina en la autoría de los artículos publicados en la RPMESP durante un periodo de 21 años. Aproximadamente, dos de cada tres publicaciones contaban con al menos una autora. Sin embargo, la proporción de artículos con mujeres en los roles privilegiados de autoría fue menor al $30 \%$. No se observaron cambios importantes entre el inicio y el final del periodo de estudio. Por otro lado, la participación femenina fue menor en equipos en los que un médico(a) ocupaba uno de los roles privilegiados de autoría y mayor cuando un autor de filiación extranjera ocupaba una de estas posiciones. Asimismo, los tiempos editoriales fueron más largos cuando mujeres ocuparon roles privilegiados de autoría y cuando había una mayor proporción de mujeres entre los autores.

De acuerdo con los resultados, las distintas formas de participación femenina en la RPMESP no han presentado cambios significativos entre el inicio y el final de periodo de estudio. Centeno-Leguía et al. evaluaron las publicaciones en las que había al menos una mujer como autora en revistas médicas peruanas indizadas a SciELO. Esta investigación tampoco halló una tendencia en el tiempo para la primera autoría o corresponsalía femenina ${ }^{(21)}$. Estos

Tabla 2. Participación femenina en artículos originales* según participación de médicos, Revista Peruana de Medicina Experimental y Salud Pública entre 1997 y $2017(n=743)$

\begin{tabular}{|c|c|c|c|c|c|c|c|c|c|c|c|c|c|c|c|}
\hline \multirow{2}{*}{$\begin{array}{l}\text { En los } \\
\text { artículos en } \\
\text { que... }\end{array}$} & \multicolumn{2}{|c|}{$\begin{array}{c}\begin{array}{c}\text { Mujer primera } \\
\text { autora }\end{array} \\
\end{array}$} & \multirow[b]{2}{*}{$\begin{array}{l}\text { Valor } \\
\text { de } p^{\ddagger}\end{array}$} & \multicolumn{2}{|c|}{$\begin{array}{c}\text { Mujer autora } \\
\text { senior }\end{array}$} & \multirow[b]{2}{*}{$\begin{array}{l}\text { Valor } \\
\text { de } \mathrm{p}^{\ddagger}\end{array}$} & \multicolumn{2}{|c|}{$\begin{array}{c}\text { Mujer } \\
\text { corresponsal }\end{array}$} & \multirow[b]{2}{*}{$\begin{array}{l}\text { Valor } \\
\text { de } \mathrm{p}^{\ddagger}\end{array}$} & \multicolumn{2}{|c|}{$\begin{array}{c}\text { Al menos } \\
\text { una autora }\end{array}$} & \multirow[b]{2}{*}{$\begin{array}{l}\text { Valor } \\
\text { de } p^{\ddagger}\end{array}$} & \multicolumn{2}{|c|}{$\begin{array}{c}\text { Proporción de } \\
\text { autoras }^{\dagger}\end{array}$} & \multirow[b]{2}{*}{$\begin{array}{l}\text { Valor } \\
\text { de } p^{\S}\end{array}$} \\
\hline & $\mathbf{n}$ & $\%$ & & $\mathbf{n}$ & $\%$ & & $\mathbf{n}$ & $\%$ & & $\mathbf{n}$ & $\%$ & & Mediana & $\mathbf{R I Q}$ & \\
\hline \multicolumn{16}{|c|}{ Un médico(a) fue el primer autor } \\
\hline Sí & 68 & 20,4 & $<0,001$ & 101 & 30,2 & 0,023 & 70 & 21,2 & $<0,001$ & 248 & 74,3 & $<0,001$ & 33 & $0-50$ & $<0,001$ \\
\hline No & 214 & 52,5 & & 156 & 38,2 & & 177 & 45,7 & & 349 & 85,5 & & 50 & $25-67$ & \\
\hline \multicolumn{16}{|c|}{ Un médico(a) fue el autor senior } \\
\hline Sí & 134 & 34,5 & 0,039 & 83 & 21,4 & $<0,001$ & 117 & 31,0 & 0,043 & 296 & 76,3 & 0,003 & 33 & $13-50$ & $<0,001$ \\
\hline No & 148 & 41,9 & & 173 & 49,0 & & 130 & 38,2 & & 300 & 85,0 & & 50 & $25-67$ & \\
\hline \multicolumn{16}{|c|}{ Un médico(a) fue el autor corresponsal } \\
\hline Sí & 80 & 23,3 & $<0,001$ & 102 & 29,7 & 0,008 & 68 & 19,8 & $<0,001$ & 256 & 74,4 & $<0,001$ & 33 & $0-50$ & $<0,001$ \\
\hline No & 189 & 50,7 & & 146 & 39,1 & & 178 & 47,7 & & 324 & 86,9 & & 50 & $30-67$ & \\
\hline
\end{tabular}

*Incluye artículos originales y originales breves.

†Proporción de autoras entre el número de total de autores del artículo.

¥Prueba de chi-cuadrado.

§Prueba de suma de rangos de Wilcoxon.

$\mathrm{RIQ}$ : rango intercuartílico. 
Tabla 3. Participación femenina en artículos originales* según participación de autores con filiación extranjera (no peruana), Revista Peruana de Medicina Experimental y Salud Pública entre 1997 y 2017 (n=743)

\begin{tabular}{|c|c|c|c|c|c|c|c|c|c|c|c|c|c|c|c|}
\hline \multirow{2}{*}{$\begin{array}{l}\text { En los } \\
\text { artículos } \\
\text { en que... }\end{array}$} & \multicolumn{2}{|c|}{$\begin{array}{l}\text { Mujer } \\
\text { primera } \\
\text { autora }\end{array}$} & \multirow[b]{2}{*}{$\begin{array}{l}\text { Valor } \\
\text { de } p^{\ddagger}\end{array}$} & \multicolumn{2}{|c|}{$\begin{array}{l}\text { Mujer } \\
\text { autora } \\
\text { senior }\end{array}$} & \multirow[b]{2}{*}{$\begin{array}{l}\text { Valor } \\
\text { de } p^{\ddagger}\end{array}$} & \multicolumn{2}{|c|}{$\begin{array}{c}\text { Mujer } \\
\text { corresponsal }\end{array}$} & \multirow[b]{2}{*}{$\begin{array}{l}\text { Valor } \\
\text { de } \mathrm{p}^{\ddagger}\end{array}$} & \multicolumn{2}{|c|}{$\begin{array}{l}\text { Al menos } \\
\text { una autora }\end{array}$} & \multirow[b]{2}{*}{$\begin{array}{l}\text { Valor } \\
\text { de } p^{\ddagger}\end{array}$} & \multicolumn{2}{|c|}{$\begin{array}{l}\text { Proporción de } \\
\text { autoras }^{\dagger}\end{array}$} & \multirow[b]{2}{*}{$\begin{array}{c}\text { Valor de } \\
\mathbf{p}^{\S}\end{array}$} \\
\hline & $\mathbf{n}$ & $\%$ & & $\mathbf{n}$ & $\%$ & & $\mathrm{n}$ & $\%$ & & $\mathbf{n}$ & $\%$ & & Mediana & $\mathbf{R I Q}$ & \\
\hline \multicolumn{16}{|c|}{ Primer autor con filiación extranjera } \\
\hline Sí & 50 & 50,1 & 0,006 & 42 & 42,4 & 0,084 & 44 & 44,4 & 0,023 & 82 & 82,8 & 0,527 & 50 & $20-55$ & 0,009 \\
\hline No & 232 & 36,0 & & 216 & 33,5 & & 203 & 32,7 & & 516 & 80,1 & & 40 & $25-67$ & \\
\hline \multicolumn{16}{|c|}{ Autor senior con filiación extranjera } \\
\hline Sí & 53 & 46,1 & 0,052 & 49 & 42,6 & 0,051 & 48 & 42,1 & 0,059 & 98 & 85,2 & 0,161 & 50 & $29-67$ & 0,005 \\
\hline No & 229 & 36,5 & & 208 & 33,2 & & 199 & 33,0 & & 499 & 79,6 & & 40 & $20-57$ & \\
\hline \multicolumn{16}{|c|}{ Autor corresponsal con filiación extranjera } \\
\hline Sí & 49 & 49,5 & 0,008 & 42 & 42,4 & 0,079 & 44 & 44,4 & 0,023 & 81 & 81,8 & 0,812 & 50 & $25-67$ & 0,016 \\
\hline No & 221 & 35,7 & & 207 & 33,4 & & 203 & 32,7 & & 501 & 80,8 & & 40 & $20-50$ & \\
\hline
\end{tabular}

*Incluye artículos originales y originales breves.

†Proporción de autoras entre el número de total de autores del artículo.

‡Prueba de chi-cuadrado.

§ Prueba de suma de rangos de Wilcoxon.

$\mathrm{RIQ}$ : rango intercuartílico.

hallazgos no son consistentes con el incremento global de la participación femenina en las publicaciones académicas ${ }^{(8-18)}$. Sin embargo, en algunos estudios se ha encontrado una desaceleración del incremento o incluso un efecto meseta al alcanzar cierta proporción de primeras autoras o autoras corresponsales ${ }^{(10,11,13,14)}$. Por otro lado, los cambios en algunas revistas no han sido tan marcados. Por ejemplo, entre 1994 y 2014, la proporción de primeras autoras en seis revistas médicas de alto impacto aumentó de $28 \%$ a $38 \%{ }^{(10)}$. Es posible que nuestros resultados se deban a que, en este periodo, no haya incrementado sustancialmente la proporción mujeres en el ámbito científico/académico en el Perú. Por otro lado, se observa una aparente disminución hacia la mitad del periodo de estudio; la cual podría ser producto de fluctuaciones aleatorias.

Aproximadamente un tercio de los artículos tuvo a una mujer en los roles privilegiados de autoría. Es difícil establecer con exactitud qué tan amplia es la brecha existente, pues necesitaríamos saber cuál fue la proporción de mujeres

Tabla 4. Tiempo de los procesos de publicación de artículos originales* según participación femenina, Revista Peruana de Medicina Experimental y Salud Pública entre 1997 y 2017 ( $n=498)$

\begin{tabular}{|c|c|c|c|c|c|c|c|c|c|}
\hline \multirow[t]{2}{*}{ Artículo } & \multicolumn{2}{|c|}{$\begin{array}{c}\text { Envío hasta } \\
\text { aceptación (días) }\end{array}$} & \multirow[b]{2}{*}{$\begin{array}{l}\text { Valor } \\
\text { de } p^{\dagger}\end{array}$} & \multicolumn{2}{|c|}{$\begin{array}{l}\text { Aceptación hasta } \\
\text { publicación (días) }\end{array}$} & \multirow[b]{2}{*}{$\begin{array}{l}\text { Valor } \\
\text { de } p^{\dagger}\end{array}$} & \multicolumn{2}{|c|}{$\begin{array}{c}\text { Envío hasta } \\
\text { publicación (días) }\end{array}$} & \multirow[b]{2}{*}{$\begin{array}{c}\text { Valor de } \\
p^{\dagger}\end{array}$} \\
\hline & Mediana & $\mathbf{R I Q}$ & & Mediana & RIQ & & Mediana & $\mathbf{R I Q}$ & \\
\hline \multicolumn{10}{|l|}{ Primer autor } \\
\hline Varón & 119 & $72-169$ & $<0,001$ & 56 & $30-105$ & 0,892 & 185 & $123-270$ & 0,002 \\
\hline Mujer & 150 & 98-198 & & 55 & $31-104$ & & 224 & $151-300$ & \\
\hline \multicolumn{10}{|l|}{ Autor senior } \\
\hline Varón & 121 & $78-175$ & 0,008 & 56 & $32-110$ & 0,342 & 196 & $129-275$ & 0,132 \\
\hline Mujer & 143 & $91-209$ & & 56 & $29-86$ & & 221 & $145-284$ & \\
\hline \multicolumn{10}{|c|}{ Autor corresponsal } \\
\hline Varón & 119 & $72-168$ & $<0,001$ & 55 & $30-106$ & 0,477 & 182 & $123-267$ & $<0,001$ \\
\hline Mujer & 151 & $103-210$ & & 59 & $34-104$ & & 227 & $157-306$ & \\
\hline \multicolumn{10}{|c|}{ Proporción de autorasł (terciles) } \\
\hline $0 \%-25 \%$ & 113 & $72-156$ & $0,001^{\S}$ & 53 & $28-110$ & $0,861 \S$ & 182 & $122-271$ & $0,005 \S$ \\
\hline$>25 \%-50 \%$ & 132 & $81-181$ & & 56 & $33-104$ & & 197 & $136-272$ & \\
\hline$>50 \%-100 \%$ & 147 & $98-218$ & & 56 & $30-103$ & & 233 & $151-300$ & \\
\hline
\end{tabular}

* Incluye artículos originales y originales breves.

† Prueba de suma de rangos de Wilcoxon.

‡ Proporción de autoras entre el número de total de autores del artículo.

$\S$ Prueba de tendencia; extensión de la suma de rangos de Wilcoxon por Cuzik.

RIQ: rango intercuartílico. 
científicas en el área biomédica en el Perú durante el periodo de estudio. Además, existen otras limitantes, como el hecho de que las científicas peruanas pueden enviar su trabajo a otras revistas o que científicas extranjeras envíen sus resultados a la RPMESP. Respecto a la proporción de científicas en el área biomédica, el Censo Nacional de Investigación y Desarrollo a Centros de Investigación 2016, llevado a cabo por el Consejo Nacional de Ciencia, Tecnología e Innovación Tecnológica (CONCYTEC) encontró que, de los 441 investigadores en Ciencias Médicas y de la Salud, 197 (44,7\%) fueron mujeres ${ }^{(22)}$. Si esta proporción de mujeres es similar en los investigadores no elegibles para el censo, es probable que su contraste con el $30 \%$ de artículos en los que mujeres ostentaron roles privilegiados de autoría suponga una brecha de género. Sin embargo, es importante tener en cuenta las limitantes ya mencionadas. Es probable que esta brecha se acorte con el recambio generacional, ya que la composición de las nuevas cohortes de científicos se caracteriza por tener proporciones similares de varones y mujeres.

La proporción de mujeres en los roles privilegiados de autoría podría también explicarse por la decisión de científicas peruanas de publicar sus resultados en revistas diferentes a la RPMESP. Existe un número importante de mujeres peruanas con alta producción científica, quienes podrían preferir publicar la mayor parte de sus artículos en revistas extranjeras o inglés. Por otro lado, puede que otras revistas peruanas reciban una mayor cantidad de artículos de científicas del país. Sería conveniente ampliar la exploración de este fenómeno en revistas indizadas a otras bases de datos como, por ejemplo, SciELO Perú.

Esta brecha podría reflejar también una disparidad en el número de artículos que son enviados y/o aceptados en la RPMESP. Es importante reconocer que no conocemos los denominadores; es decir, el número de artículos enviados a la revista. Varios escenarios podrían explicar estas diferencias: a) la proporción de artículos enviados que, finalmente, se publica no depende de si los roles privilegiados de autoría son ocupados por varones o mujeres (lo cual supondría que, en promedio, menos artículos son sometidos por mujeres); b) los artículos con varones en roles privilegiados de autoría son aceptados en mayor proporción que los artículos con mujeres en estas posiciones; o c) una combinación de ambos escenarios. Sin embargo, nuestro estudio no permite determinar ninguna potencial explicación, pues no se evaluaron los artículos sometidos a la revista.

Con respecto al primer escenario, un estudio estadounidense encontró que las médicas que se desempeñan en la academia publican, en promedio, una menor cantidad de artículos que sus contrapartes varones. Los autores atribuyen estas diferencias a la desproporcionada carga familiar que las mujeres frecuentemente asumen, la no disponibilidad de adecuada mentoría científica, entre otros ${ }^{(23)}$. El segundo escenario supondría que los artículos sometidos a la revista por mujeres son rechazados en mayor proporción que los artículos sometidos por hombres. Un estudio austriaco encontró que los artículos con una mujer como primera autora tenían mayor probabilidad de ser rechazados rápidamente, pero no hubo diferencias en la proporción general de rechazos ${ }^{(24)}$. En la misma línea, varios estudios sugieren la existencia de sesgos editoriales de género, los cuales devienen en una menor cantidad de mujeres como pares revisores de artículos ${ }^{(24,25)}$ y como miembros de comités editoriales ${ }^{(26,27)}$. Sin embargo, los resultados no permiten determinar cuál de los escenarios anteriormente planteados explica los hallazgos.

Si una mujer ocupaba uno de los roles privilegiados de la autoría, los procesos editoriales, en promedio, demoraron entre 20 a 45 días más en comparación con los artículos donde un varón ocupaba estas posiciones. Los tiempos editoriales incluyen la revisión del artículo por pares expertos, quienes son ciegos a la identidad del autor, por lo cual es poco probable que el tiempo de este proceso explique las diferencias entre varones y mujeres. El tiempo que los autores toman para el levantamiento de observaciones de los revisores podría explicarlas. Las mujeres suelen asumir mayor responsabilidad familiar ${ }^{(23,28)}$, lo cual podría competir con el tiempo disponible para trabajar en el levantamiento de observaciones. Por otro lado, en caso de que existiera sesgo editorial, es posible que exista confusión con la etapa de carrera de los autores. Bajo el supuesto de que había más varones en generaciones anteriores de científicos, podría haber cierta tendencia favorable hacia el trabajo sometido por autores con largas trayectorias profesionales en investigación.

En los artículos originales en los que un médico es primer autor, autor senior o autor corresponsal, es menos probable que una mujer ocupe alguno de los roles privilegiados de autoría, menos probable que haya al menos una mujer en la lista de autores y que la proporción de autoras sea más baja. Esto podría deberse a que, si bien existe una mayor representación femenina en las nuevas generaciones de médicos, esta es todavía muy reciente para cambiar la proporción general de mujeres en el cuerpo médico actual. Según el Instituto Nacional de Estadística e Informática y la Superintendencia Nacional de Salud, al 2016, 24,5\% de los médicos que trabajan en el sistema de salud peruano son mujeres ${ }^{(29)}$. Por otro lado, reportes norteamericanos concuerdan en que, a pesar de que el número de mujeres en medicina ha aumentado ${ }^{(1)}$, aún existe una brecha importante en la ocupación de posiciones de liderazgo ${ }^{(1,28)}$. Según Rochon et al., esto puede deberse a las responsabilidades familiares asumidas por las mujeres, a una mayor iniciativa de los varones o por sesgos de género, que podrían llevar a dar preferencia a los varones ${ }^{(28)}$.

Por el contrario, en los artículos de autores con filiación extranjera, es más probable encontrar a una mujer en los roles privilegiados, así como una mayor proporción de mujeres en la lista de autores. Nuestros resultados sugieren 
que, en comparación con equipos de investigación del extranjero, los equipos peruanos aún no cuentan con representación paritaria de mujeres y que ellas no están ocupando las posiciones de liderazgo en estos equipos. En vista de que la mayoría de investigadores que envía sus artículos a la RPMESP proviene de países latinoamericanos ${ }^{(30)}$, es probable que este resultado sea principalmente un reflejo de la realidad nacional en comparación a la realidad regional. También, es probable que haya mayor diferencia con artículos de autores del hemisferio norte. Nuestros resultados podrían deberse a una menor proporción de mujeres científicas en el Perú. Sin embargo, podría ser, también, un indicador de que las condiciones de trabajo en ciencia para las mujeres en nuestro país.

En nuestro país, CONCYTEC ha planteado propuestas para incentivar y empoderar a las mujeres. Una de ellas fue la instalación del Comité Pro-Mujer, constituido por investigadoras peruanas de reconocida trayectoria. Este busca impulsar la participación femenina a través deacciones concretas que promuevan igualdad de oportunidades para las mujeres ${ }^{(31)}$. Otra iniciativa importante son los concursos de fondos de investigación dirigidos específicamente a promover la participación de la mujer, en el que se otorga $5 \%$ adicional si la investigadora principal es mujer o si más del $50 \%$ de investigadoras son mujeres ${ }^{(32)}$. Se ha identificado que la falta de modelos a seguir es un factor importante en la academia ${ }^{(23,28,33)}$. En ese sentido, las universidades podrían incrementar la visualización de las mujeres investigadoras, así como proveer tiempo protegido para actividades entre mentoras e investigadoras en formación. Por otro lado, brindar flexibilidad para el trabajo desde casa o las licencias de paternidad compartida podrían contribuir a aligerar carga familiar de la mujer y mejorar su balance entre familia y trabajo ${ }^{(33)}$.

Nuestro estudio presenta ciertas limitaciones. Primero, la evaluación del género no fue por consulta directa con los participantes sino por inspección del nombre. Por otro lado, no hemos controlado el potencial efecto confusor de otras características de los autores (grado académico, etapa de carrera, especialidad en el caso de medicina, entre otras) en las comparaciones hechas. Adicionalmente, cabe recalcar que los resultados de esta investigación sólo representan la realidad de la RPMESP y no la participación de la mujer en publicaciones científicas en general en el Perú.
En conclusión, en menos de un tercio de los artículos publicados en la RPMESP entre 1997 y 2017, los roles de primer autor, autor senior y autor corresponsal fueron ocupados por una mujer. Si bien se encuentran ligeras fluctuaciones de la participación femenina en el periodo de estudio; no existen cambios considerables entre el inicio y el final de este. La participación femenina en la autoría fue menor si quien ocupaba los roles privilegiados de autoría era un médico(a) y mayor si quien ocupaba estas posiciones era un autor de filiación extranjera. Por último, los tiempos editoriales fueron mayores para los artículos con mujeres en los roles privilegiados de autoría y para artículos con mayor proporción de mujeres.

Nuestros resultados muestran que aún existen brechas y que, aparentemente, estas no han mejorado en los últimos 20 años. Como corolario, nos gustaría aclarar que nuestro artículo no es una crítica directa a la RPMESP ni a su Comité Editorial. Por el contrario, preferiríamos que sea tomado como una (auto)crítica a la comunidad científica en su conjunto. Sin ir muy lejos, de los tres autores de este artículo, solo uno es una mujer, quien no ocupa ninguno de los roles privilegiados de autoría. Si bien esto se decidió en forma consensuada y justa, debemos preguntarnos si estamos omitiendo acciones para derribar las barreras que limitan la participación y liderazgo de la mujer en el ámbito científico-académico. Invitamos a los lectores a reflexionar sobre este punto y, de ser necesario, tomar acción a nivel proximal, a nivel de nuestros propios equipos de investigación.

Recomendamos que se realice posterior investigación, cuantitativa y cualitativa, que ayude conocer las razones que existen detrás de nuestros hallazgos para así poder planear intervenciones estructurales dirigidas hacia un mundo de ciencia más equitativo para todas y todos los investigadores.

Contribuciones de los autores: RPE concibió el estudio. DGA, IHM y RPE lo diseñaron. DGA e IHM recolectaron los datos. RPE realizó el análisis estadístico. DGA e IHM redactaron la versión preliminar del manuscrito y RPE la revisó y editó. DGA, IHM y RPE aceptan la versión del manuscrito enviada para publicación y se hacen responsables por su contenido.

Fuentes de financiamiento: autofinanciado.

Conflictos de interés: ninguno que declarar.

\section{REFERENCIAS BIBLIOGRÁFICAS}

1. Association of American Medical Colleges. The state of women in academic medicine: The pipeline and pathways to leadership [Internet]. Washington: AAMC; 2014 [citado el 01 de septiembre de 2019]. Disponible en: https://store. aamc.org/downloadable/download/link/
id/MC4wMzY5NTIwMCAxNTY3MTAyODg1MjQ5NTMxMjk2NzE5OTkw/

2. Ramakrishnan A, Sambuco D, Jagsi R. Women's participation in the medical profession: insights from experiences in Japan, Scandinavia, Russia, and
Eastern Europe. J Womens Health. 2014; 23(11):927-34. doi: 10.1089/ jwh.2014.4736.

3. Russo G, Gonçalves L, Craveiro I,Dussault G. Feminization of the medical workforce in low-income settings; findings from surveys in three African capital cities. Hum 
Resour Health. 2015;13:64. doi: 10.1186/ s12960-015-0064-9.

4. Ng-Sueng LF, Vargas-Matos I, MaytaTristán P, Pereyra-Elías R, MontenegroIdrogo JJ, Inga-Berrospi F, et al. Gender Associated with the Intention to Choose a Medical Specialty in Medical Students: A Cross-Sectional Study in 11 Countries in Latin America. PLoS One. 2016;11(8):e0161000. doi: 10.1371/ journal.pone.0161000.

5. CaterSW, Yoon SC, Lowell DA, Campbell JC, Sulioti G, Qin R, et al. Bridging the Gap: Identifying Global Trends in Gender Disparity Among the Radiology Physician Workforce. Acad Radiol. 2018;25(8):1052-1061. doi: 10.1016/j. acra.2017.12.021.

6. Jena AB, Khullar D, Ho O, Olenski AR, Blumenthal DM. Sex Differences in Academic Rank in US Medical Schools in 2014.JAMA. 2015;314(11):1149-58. doi: 10.1001/jama.2015.10680.

7. Jena AB, Olenski AR, Blumenthal DM. Sex Differences in Physician Salary in US Public Medical Schools. JAMA Intern Med. 2016;176(9):1294-304. doi: 10.1001/jamainternmed.2016.3284.

8. Jagsi R, Guancial EA, Worobey CC, Henault LE, Chang Y, Starr R, et al. The "gender gap" in authorship of academic medical literature-a 35-year perspective. N Engl J Med. 2006;355(3):281-7.

9. Sidhu R, Rajashekhar P, Lavin VL, Parry J, Attwood J, Holdcroft A, et al. The gender imbalance in academic medicine: a study of female authorship in the United Kingdom. J R Soc Med. 2009;102(8):337-42.

10. Filardo G, da Graca B, Sass DM, Pollock BD, Smith EB, Martinez MA. Trends and comparisons of female first authorship in high impact medical journals: observational study (1994-2014). BMJ. 2016;352:i847.

11. Fishman M, Williams WA 2nd, Goodman DM, Ross LF. Gender Differences in the Authorship of Original Research in Pediatric Journals, 2001-2016. J Pediatr. 2017;191:244-249.e1.

12. Ouyang D, Sing D, Shah S, Hu J, Duvernoy C, Harrington RA, et al. Sex Disparities in Authorship Order of Cardiology Scientific Publications. Circ Cardiovasc Qual Outcomes. 2018;11(12):e005040.

13. Sing DC, Jain D, Ouyang D. Gender trends in authorship of spine-related academic literature-a 39-year perspective. Spine J. 2017;17(11):1749-1754.

14. Süßenbacher S, Amering M, Gmeiner A, Schrank B. Gender-gaps and glass ceilings: A survey of gender-specific publication trends in Psychiatry between 1994 and 2014. Eur Psychiatry. 2017;44:90-95.

15. Bhattacharyya N, Shapiro NL. Increased female authorship in otolargyngology over the past three decades. Laryngoscope. 2000; 110:358-61.

16. Feramisco JD, Leitenberger JJ, Redfern SI, Bian A, Xie XJ, Resneck JS Jr. A gender gap in the dermatology literature? Cross-sectional analysis of manuscript authorship trends in dermatology journals during 3 decades. J Am Acad Dermatol. 2009;60(1):63-9.

17. Mimouni M, Zayit-Soudry S, Segal O, Barak Y, Nemet AY, Shulman S, et al. Trends in Authorship of Articles in Major Ophthalmology Journals by Gender, 2002-2014. Ophthalmology. 2016;123(8):1824-8.

18. Silvestre J, Wu LC, Lin IC, Serletti JM. Gender Authorship Trends of Plastic Surgery Research in the United States. Plast Reconstr Surg. 2016;138(1):136e-42e.

19. Burstein Z, Mayta-Tristán P. La Revista Peruana de Medicina Experimental y Salud Pública ahora indizada en MEDLINE/ Index Medicus. Rev Peru Med Exp Salud Publica. 2010;27(3):312-4.

20. Huamaní C. Análisis de la producción, visibilidad y citación de la Revista Peruana de Medicina Experimental y Salud Pública, 2002-2009. Rev Peru Med Exp Salud Publica. 2010;27(3):367-72.

21. Centeno-Leguía D, Morales-Concha L, Lopez CE, Mejia CR. Mujeres científicas: características y factores asociados a la primera autoría y corresponsalía en revistas peruanas indizadas a SciELO, 2010-2015. Educ Med. 2018 [Pre-publicación online]

22. Consejo Nacional de Ciencia, Tecnología e Innovación Tecnológica. I Censo Nacional de Investigación y Desarrollo a Centros de Investigación 2016. Lima: CONCYTEC; 2017. [citado el 01 de septiembre de 2019]. Disponible en: http://portal.concytec. gob.pe/index.php/publicaciones/censonacional-id

23. RajA, Carr PL, Kaplan SE, Terrin N, Breeze JL, Freund KM. Longitudinal Analysis of Gender Differences in Academic Productivity Among Medical Faculty Across 24 Medical Schools in the United States. Acad Med. 2016;91(8):1074-9.

24. Heckenberg A, Druml C. Gender aspects in medical publication - the Wiener klinische Wochenschrift. Wien Klin Wochenschr. 2010;122(5-6):141-5.

25. Lerback J, Hanson B. Journals invite too few women to referee. Nature. 2017; 541: 455-57.
26. Dickersin K, Fredman L, Flegal KM, Scott JD, Crawley B. Is there a sex bias in choosing editors? Epidemiology journals as an example. JAMA. 1998;280:260-4.

27. Ioannidou E, Rosania A. Underrepresentation of women on dental journal editorial boards. PLoS One. 2015; 10: 1-9.

28. Rochon PA, Davidoff F, Levinson W. Women in academic medicine leadership: has anything changed in 25 years?. Acad Med. 2016; 91: 1053-56.

29. Instituto Nacional de Estadística e Informática. Informe final: Encuesta Nacional de Satisfacción de Usuarios en Salud 2016. Lima: INEI; 2016. [citado el 01 de septiembre de 2019]. Disponible en: http://portal.susalud.gob.pe/wpcontent/uploads/archivo/encuestasat-nac/2016/INFORME_FINAL_ ENSUSALUD_2016.pdf

30. Burstein Z, Alarco JJ. La Revista Peruana de Medicina Experimental y Salud Pública asume con propósitos renovados sus responsabilidades para el 2019. Rev Peru Med Exp Salud Publica. 2019;36(1):5-6.

31. Consejo Nacional de Ciencia, Tecnología e Innovación Tecnológica. Concytec instaló Comité Pro Mujer en CTI integrado por 10 destacadas investigadoras. Lima: CONCYTEC; 2019. [citado el 01 de septiembre de 2019]. Disponible en: https://portal.concytec.gob.pe/index. $\mathrm{php} /$ noticias/1746-concytec-instalocomite-pro-mujer-en-cti-integrado-por10-destacadas-cientificas

32. Fondo Nacional de Desarrollo Científico, Tecnológico y de Innovación Tecnológica. Concursos De Investigación Aplicada Y Desarrollo Tecnológico Promueven La Participación De Regiones, Mujeres Y Jóvenes. Lima: FONDECYT; 2018. [citado el 01 de septiembre de 2019]. Disponible en: http://www.fondecyt. gob.pe/fondecyt-informa/concursosde-investigacion-aplicada-y-desarrollotecnologico-promueven-la-participacionde-regiones-mujeres-y-jovenes

33. D’Armiento J, Witte SS, Dutt K, Wall M, McAllister G; Columbia University Senate Commission on the Status of Women. Achieving women's equity in academic medicine: challenging the standards. Lancet. 2019;393(10171):e15-e16.

\section{Correspondencia: Reneé Pereyra-Elías}

Escuela de Medicina, Universidad Peruana de Ciencias Aplicadas, Lima, Perú.

Dirección: Alameda San Marcos cuadra 2, Villa (Chorrillos). Lima, Perú.

Email:renee.pereyra.elias@gmail.com 